\title{
Radiotherapy as a Cure for Covid-19 Pneumonia: Is this a Possibility?
}

\author{
Partha Pratim Medhi, Shashank Bansal, Faridha Jane Momin, Ghritashee Bora
}

Department of Radiation Oncology, Dr. B.Borooah Cancer Institute, Guwahati, ASSAM, India.

\begin{abstract}
The ongoing pandemic of COVID-19 caused by the Severe Acute Respiratory Syndrome Corona virus 2 (SARS-CoV-2) has affected the healthcare system worldwide to a great extent. Millions of people have succumbed to the disease which might vary from mild febrile illness to severe Acute Lung Injury ultimately resulting in ARDS (Acute Respiratory Distress Syndrome). The mechanism of lung pathology due to SARS $\mathrm{CoV}-2$ infection though unclear, is thought to be due to an imbalance in the inflammatory cascade; leading to a condition called Chemokine Release Syndrome. Low dose Radiotherapy which was historically used to treat Pneumonia, can serve as an approach for this subset of patients; by virtue of its anti-inflammatory response. Thus, in the present time, where a perfect cure is yet to derived all possible interventions should be looked upon with a ray of hope.
\end{abstract}

Keywords: SARS-CoV-2-COVID-19-Pneumonia-Radiation Therapy

Asian Pac J Cancer Care, 5 (Suppl 1), 157-159

\section{Introduction}

The COVID-19 pandemic has paralysed the health care system of the world, by affecting millions of people till date. It is caused by the Severe Acute Respiratory Syndrome Corona virus 2 (SARS-CoV-2). A spectrum of symptoms can be attributed to it, starting from mild flu-like symptoms to severe lung injury or Pneumonia [1]. Approximately $20 \%$ of patients progress to ARDS , which often results in death [2].

\section{Immune mechanism involved}

SARS-CoV infection activates both the innate and adaptive immune mechanism. In response to the infection, macrophages release various pro-inflammatory cytokines like IL 1,IL 6, TNF $\alpha$, TGF $\beta$ etc leading to a condition called Cytokine Release Syndrome [3]. In addition the infected alveolar epithelial cells also produce various Chemokines like CCL 2,CXCL-10, CXCL-9 [4]. This dysregulated release of cytokines and chemokines cause an imbalance in the immune mechanism, thus playing a role in the pathogenesis of the disease.

This again results in enhanced endothelial and epithelial cell apoptosis, increased vascular leakage,
Submission Date: 05/30/2020Ａcceptance Date: 06/28/2020 suboptimal response to anti-body and decreased virus clearance [5-6].

Importance of Radiation and its role in Pneumonia

Radiation induces cell death by destruction of DNA either directly or indirectly by production of free radicals (ions) inside the body. Historically, X-rays have been used to treat cancer since Emil Herman Grubbe treated the first patient in 1896. Benign intracranial tumours (e.g. Schwannoma, Craniopharyngioma etc), Glomus tumors, Juvenile Nasopharyngeal Angiofibroma and many other benign tumors are also treated by radiotherapy. Interestingly, there are reports of use of low dose radiation treatment in many acute inflammatory processes and infectious diseases such as gas gangrene, carbuncles, sinusitis, arthritis and mastitis in the pre-antibiotic era with significant cure rates. Even today, low dose radiation is prescribed for benign painful chronic inflammatory degenerative disorders such as periarthritis in Germany. Experiments over past three decades have revealed a multilevel interrelationship between low-dose ionizing radiation and inflammatory cascades which include:

Corresponding Author:

Dr. Ghritashee Bora

Department of Radiation Oncology, Dr. B.Borooah Cancer Institute, Guwahati, ASSAM, India.

Email: ghrita123@gmail.com 
modulation of the inflammatory properties of leukocytes, macrophages, fifibroblasts, and endothelial cells, as well as of the secretion of cytokines/chemokines and growth factors [7].

In the early 20th century, Pneumonia was one of the dreaded diseases with mortality upto $40 \%$ even in treated cases. Before the advent of widespread use of antibiotics, Serum therapy was the only effective treatment for pneumonia, but it was expensive and carried a high risk of anaphylactic reactions. This led to the exploration of Radiotherapy as an alternative curative option for pneumonia in the 1930s. The hypothesis was that X-ray treatment would increase the metabolic digestion of the exudative material,leading to a resolution of the pneumonia.Calabrese and Dhawan [8] have compiled a review of all the reported studies on this topic from the United States. Between 1905-1946, fifteen studies showed that over 800 patients of bacterial, viral and atypical pneumonia were effectively treated by low dose kilovoltage X-rays. All these studies reported significant improvement in clinical symptoms within hours of initiation of therapy and decrease in mortality incidence to less than $10 \%$ in their patients which was comparable to serum therapy and sulphonamide treatment during the same time period. Irrespective of the age, health status, etiology and other diverse clinical settings, these researchers consistently reported the benefit of a single low dose X-ray exposure to effectively reverse the course of pneumonia. Benefits were uniformly better when radiotherapy was instituted in the initial stages of the disease. The target volume comprised of bilateral whole lungs and doses in the range of 20 to few hundred Roentgen were used, which given the attenuation through chest wall would likely have resulted in mean lung doses of less than 100 cGy range. The treatment became so popular that at one point of time some hospitals in US routinely treated pneumonia patients with therapeutic X-rays before admitting them for further treatment. However, with the emergence of the wonder drug Penicillin, the interest in this form of treatment for pneumonia gradually faded and there has not been a single published report in this aspect for over 70 years now.

The global pandemic of novel corona virus disease has once again challenged the medical knowledge and utility of existing therapies in preventing death from this form of community acquired pneumonia. In the absence of definitive treatment against SARS-CoV-2, clinicians have now turned to therapies with anecdotal evidence- the widespread use of Hydroxy-chloroquine for example. This scenario has once again aroused the interest of some radiation oncologists of the present era to revisit the century old hypothesis of low dose X-ray therapy in pneumonia as a potential treatment of COVID-19.

\section{Possible mechanism of Radiation damage}

Radiation can reduce inflammation by various mechanisms including induction of apoptosis by immune mediated cells, secretion of anti-inflammatory factors and reducing activity of macrophages [9]. The most accepted mechanism is via the 'Polarization of macrophages' to an anti-inflammatory or M2 phenotype. The M2 phenotype tends to suppress the over-action of the immune system whereas the M1 type over stimulates the immune system leading to Cytokine Storm [10 ].

\section{Radiation as a therapeutic approach for Pneumonia}

In a article published in Radiotherapy and Oncology journal in April, 2020, Kirkby and McKenzie [11] have called for a clinical trial to investigate the efficacy of whole lung low dose radiotherapy as potential treatment of COVID-19 pneumonia. They opine that a single fraction 30 to 100 cGy X-ray treatment could be easily delivered on a conventional megavoltage radiation therapy unit and at such low doses, common radiotherapy toxicities would not be of much concern. They believe it would present a very low risk to COVID-19 pneumonia patients, have the potential to reduce mortality and mitigate COVID-19 related burden on healthcare systems.

However, the astounding results of the studies of early twentieth century must be taken with a pinch of salt before we jump to conclusions. All the 15 reported studies by Calabrese et al [8] are case series of small number of patients, without randomization of subjects, no definite control groups for comparison or blinding of investigators, making their findings largely redundant in modern day practices of evidence based medicine. Only 2 out of the 15 studies quoted by Calabrese et al. dealt with viral pneumonia and SARS-CoV-2 being a positive-sense single-stranded RNA virus which still remains a mystery, whether the radiobiology of DNA damage applies for it, is also debatable. Add to that the complicated nature of planning and delivering bilateral whole lung radiation which comes with an inherent risk of cardiac toxicities and secondary malignancies. In today's era of precision and conformal radiotherapy, we are afraid, such an idea may find no supporters. Lastly, for a country like India where radiotherapy resources are already overburdened with existing cancer patients, incorporating COVID-19 patients into radiotherapy treatment facilities will be a herculean challenge.

In conclusion, although the idea of using radiotherapy as a potential cure for COVID-19 patients seems far-fetched, we believe its not entirely devoid of merit. The COVID-19 pandemic worldwide has cost millions of lives, totally destroying the healthcare facilities of most developed of nations. Hence all possibilities of intervention even with a glimpse of hope should be looked into. If ever there was a time to explore radiation as treatment for pneumonia on a clinically useful setting, we believe it is now.

\section{References}

1. Peiris J, Chu C, Cheng V, Chan K, Hung I, Poon L, Law K, Tang B, Hon T, Chan C, Chan K, Ng J, Zheng B, Ng W, Lai R, Guan Y, Yuen K. Clinical progression and viral load in a community outbreak of coronavirus-associated SARS pneumonia: a prospective study. The Lancet. 2003 05;361(9371):1767-1772. https://doi.org/10.1016/s01406736(03)13412-5

2. Peiris JSM, Guan Y, Yuen KY. Severe acute respiratory syndrome. Nature Medicine. 2004 Nov 30;10(S12):S88-S97. 
https://doi.org/10.1038/nm1143

3. Cheung CY, Poon LLM, Ng IHY, Luk W, Sia S, Wu MHS, Chan K, Yuen K, Gordon S, Guan Y, Peiris JSM. Cytokine Responses in Severe Acute Respiratory Syndrome Coronavirus-Infected Macrophages In Vitro: Possible Relevance to Pathogenesis. Journal of Virology. 200506 15;79(12):7819-7826. https://doi.org/10.1128/ jvi.79.12.7819-7826.2005

4. Law HKW, Cheung CY, Ng HY, Sia SF, Chan YO, Luk W, Nicholls JM, Peiris JSM, Lau YL. Chemokine up-regulation in SARS-coronavirus-infected, monocyte-derived human dendritic cells. Blood. 2005 Oct 01;106(7):2366-2374. https://doi.org/10.1182/blood-2004-10-4166

5. Rodrigue-Gervais I, Labbé K, Dagenais M, Dupaul-Chicoine J, Champagne C, Morizot A, Skeldon A, Brincks E, Vidal S, Griffith T, Saleh M. Cellular Inhibitor of Apoptosis Protein cIAP2 Protects against Pulmonary Tissue Necrosis during Influenza Virus Infection to Promote Host Survival. Cell Host \& Microbe. 2014 01;15(1):23-35. https://doi. org/10.1016/j.chom.2013.12.003

6. Zhao J, Zhao J, Perlman S. T Cell Responses Are Required for Protection from Clinical Disease and for Virus Clearance in Severe Acute Respiratory Syndrome Coronavirus-Infected Mice. Journal of Virology. 201007 07;84(18):9318-9325. https://doi.org/10.1128/jvi.01049-10

7. Rödel F, Frey B, Manda K, Hildebrandt G, Hehlgans S, Keilholz L, Seegenschmiedt MH, Gaipl US, Rödel C. Immunomodulatory Properties and Molecular Effects in Inflammatory Diseases of Low-Dose X-Irradiation. Frontiers in Oncology. 2012;2. https://doi.org/10.3389/ fonc. 2012.00120

8. Calabrese E, Dhawan G. How radiotherapy was historically used to treat pneumonia: could it be useful today?. Yale J Biol Med. 2013;86(4):555-70.

9. Arenas M, Sabater S, Hernández V, Rovirosa A, Lara P, Biete A, Panés J. Anti-inflammatory effects of low-dose radiotherapy. Strahlentherapie und Onkologie. 201208 22;188(11):975-981. https://doi.org/10.1007/s00066-012$0170-8$

10. Deloch L, Fuchs J, Rückert M, Fietkau R, Frey B, Gaipl US. Low-Dose Irradiation Differentially Impacts Macrophage Phenotype in Dependence of Fibroblast-Like Synoviocytes and Radiation Dose. Journal of Immunology Research. 2019 08 14;2019:1-11. https://doi.org/10.1155/2019/3161750

11. Kirkby C, Mackenzie M. Is low dose radiation therapy a potential treatment for COVID-19 pneumonia? Radiotherapy and Oncology. 2020 04; https://doi. org/10.1016/j.radonc.2020.04.004 